\title{
Intracranial Hemorrhage Associated with Sildenafil Use: A Very Rare Complication
}

\author{
GUNTEL Murat ', CAN Hakan, GOK Fatma, TANGULEC Goncagul, BALIKEL Unal and USTA \\ Busra Nur
}

Department of Neurology, Hatay Mustafa Kemal University, Antakya, Turkey

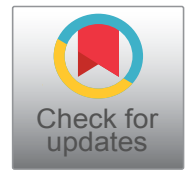

*Corresponding author: Guntel Murat, Department of Neurology, Hatay Mustafa Kemal University, Antakya, Turkey

\begin{abstract}
Intracerebral hemorrhage (ICH) (International Classification of Diseases, $9^{\text {th }}$ Revision) is a neurological deficit documented by brain CT or MRI showing the presence of an intracranial bleed in the parenchyma of the brain. $\mathrm{ICH}$ is a serious cerebrovascular condition associated with high mortality and morbidity in adults It can be classified as primary and secondary according to etiology. Primary intracranial hemorrhage often develops due to hypertension or amyloid angiopathy. Secondary intracranial hemorrhage causes are often tumor, aneurysm and vascular anomalies. Trauma is the most common reason of seconder ICH. Sildenafil citrate is a potent vasodilator agent used in the treatment of erectile dysfunction. Among its side effects, intracranial hemorrhage is a very rare complication. We present a case of a 54-year-old patient who was brought to the emergency department due to nausea, vomiting and confusion after the use of sildenafil. The patient was diagnosed with intracranial hemorrhage in the setting of sildenafil use after excluding other causes.
\end{abstract}

\section{Keywords}

Sildenafil citrate, Intracranial Hemorrhage, Nitric Oxide

\section{Introduction}

Sildenafil citrate is the first effective orally used drug for the treatment of erectile dysfunction (ED). Penis erection occurs with increased blood flow as a result of smooth muscle relaxation in the corpus cavernosum sinusoids. Nitric oxide released by sexual stimulation increases guanylate cyclase activation and causes synthesis of cyclic guanosine monophosphate (cGMP). cGMP provides smooth muscle relaxation, resulting in increased arterial flow and corporal veno-occlusion. Intracellular cGMP is destroyed by phosphodiesterase enzymes. Although four phosphodiesterase isoforms (type $2,3,4,5$ ) are detected in human penile tissue, the predominant isoform in this tissue is type 5 . Sildenafil is an oral, potent and selective inhibitor of cGMP-specific phosphodiesterase type 5 (PDE5). Sildenafil increases the cGMP concentration in corporeal smooth muscles with this effect and strengthens the relaxant effect of nitric oxide. Sildenafil is 10-10,000 times more selective on PDE 5 than other phosphodiesterase isoforms. Numerous side effects of sildenafil have been reported since its use. On the contrary, in the literature, intracranial hemorrhage associated with sildenafil use is extremely rare. We wanted to present a patient with intracranial hemorrhage in life-threatening anatomical localizations in the setting of sildenafil use. This patient had no risk factors for intracranial hemorrhage.

\section{Case Report}

A 54-year-old male patient was admitted to the emergency department due to a loss of consciousness. According to the anamnesis taken from his wife, about 3-4 hours earlier, dizziness, nausea and vomiting started suddenly while chatting with her. Then the patient developed confusion and had difficulty understanding what others are saying. Finally, he was brought to the emergency department after the patient lost consciousness. According to information received from his wife, he received 2 sildenafil tablets $(100 \mathrm{mg}) 3$ hours before the complaints started, to attempt sexual intercourse, but he could not. It was learned that there was no history of sildenafil use previously but he had an erection problem recently and It was prescribed by the urologist, to whom he applied on the recommendation of his wife.

Citation: Murat G, Hakan C, Fatma G, Goncagul T, Unal B, et al. (2020) Intracranial Hemorrhage Associated with Sildenafil Use: A Very Rare Complication. Int J Neurol Neurother 7:099. doi.org/10.23937/23783001/1410099

Accepted: April 27, 2020: Published: April 29, 2020

Copyright: (C) 2020 Murat G, et al. This is an open-access article distributed under the terms of the Creative Commons Attribution License, which permits unrestricted use, distribution, and reproduction in any medium, provided the original author and source are credited. 
The patient had been normotensive and non-diabetic throughout his life. He had no hyperlipidemia. There was no drug use. He did not smoke, drink, or use illicit drugs. There was no drug addiction in the story taken from his family (cocaine etc.). His height was 1.77 meters and his weight was $81 \mathrm{~kg}$. He had no family history of cerebral arteriovenous malformation, cerebral aneurysm or intracranial hemorrhage The neurological examination at presentation was as follows: Blood pressure was 130/85, heart rate was 104 and his fever was 37.1 The patient was confused, extension response to pain and making incomprehensible sounds. The Glasgow Coma Score was 6. Direct and indirect pupillary light reflexes were absent and he had small pupils, which are both $\sim 1.5 \mathrm{~mm}$ bilaterally. There is no eye deviation. No asymmetry was observed in muscle strength and tonus examination. Reflexes are $2+$ and symmetric at the biceps, triceps, knees, and ankles. There is no response to plantar reflex bilaterally. His breath sounds were bilaterally rough in auscultation. There was no significant feature in laboratory tests. Platelet count and coagulation factors were normal. Urine toxicological examination was not performed. Afterward, the patient developed respiratory distress in the emergency department and he was intubated. Then he was transferred to the neurology intensive care unit. Cranial computed tomography (CT) scan revealed a hyperdense hematoma area with vasogenic edema was detected. It was determined that the hematoma started from basal ganglia level and spread to mesencephalon and pons level inferior and to the $4^{\text {th }}$ ventricle and pontocerebellar level in the posterior (Figure 1).

$\mathrm{ICH}$ secondary to sildenafil is a diagnosis of exclusion. The cranial CT angiography did not reveal any vascular anomalies or other reasons explaining the cause of $\mathrm{ICH}$. In the medical history, we learned that the patient had no family history of cerebral arteriovenous malformation, cerebral aneurysm or intracranial hemorrhage.

Mannitol was started as an antiedema treatment. The patient developed acute renal failure on the $5^{\text {th }}$ day of hospitalization and he was consulted to the nephrology department. Mannitol stopped and he was started receiving dialysis. Although inotropic support was started for the patient whose blood pressure decreased on the $12^{\text {th }}$ day of his hospitalization, the patient died. We suggested an autopsy to clarify the etiology, but the relatives did not accept.

\section{Discussion}

Sildenafil citrate is the first effective orally used drug for the treatment of erectile dysfunction (ED). It improves impaired erectile function by increasing blood
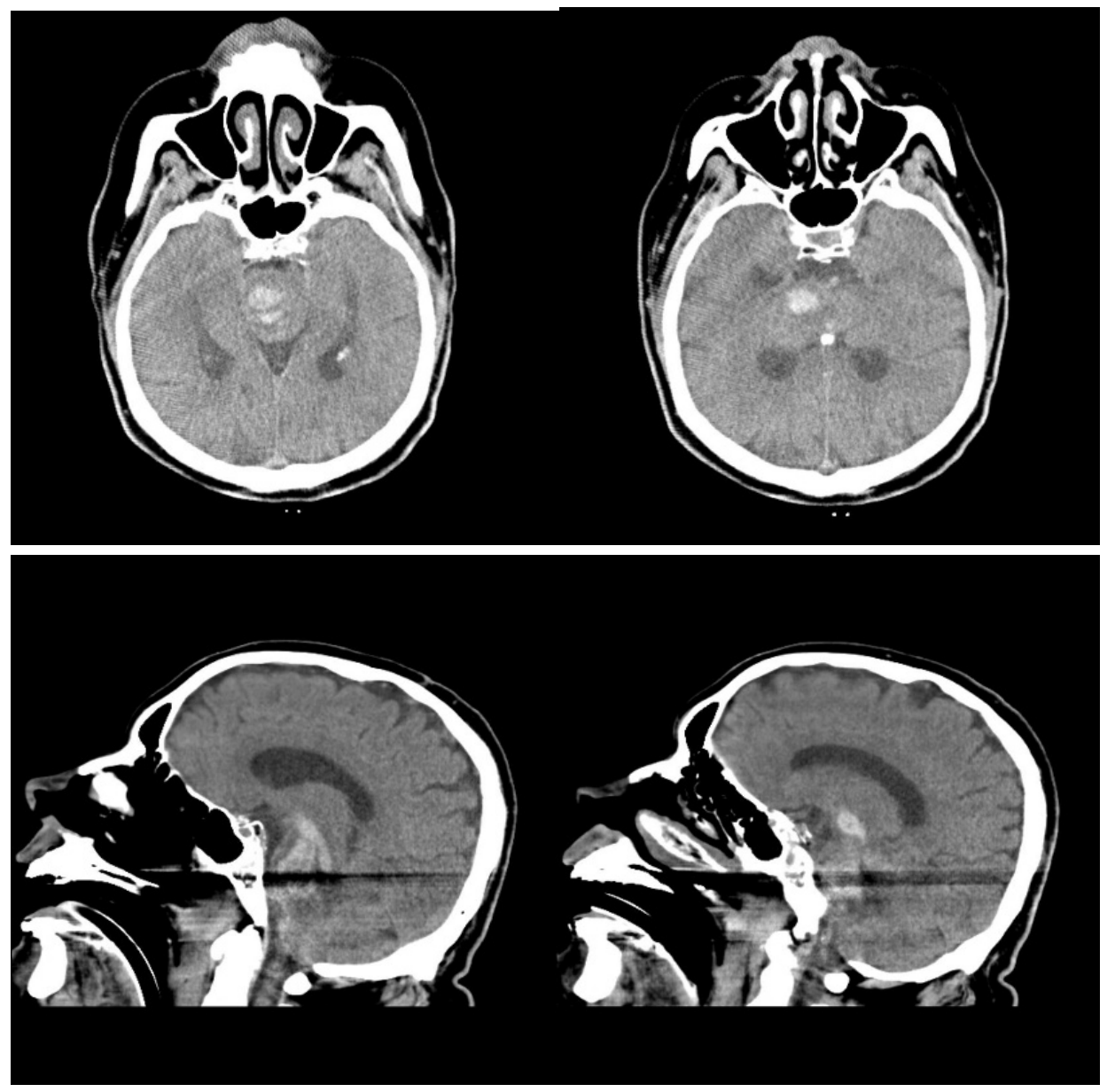

Figure 1: Computed tomography (CT) scan and lateral section lof cranium for hyperdense hematoma area with vasogenic edema. 
flow to the penis as a result of a natural response to sexual stimulation. The physiological mechanism that provides the erection of the penis requires the release of nitric oxide (NO) from the corpus cavernosum during sexual stimulation. Sildenafil does not have a direct relaxant effect on the isolated human corpus cavernosum, but it has the potential to increase the relaxant effect of nitric oxide present on this tissue. As with sexual stimulation, when the NO/cGMP pathway is activated, sildenafil inhibits the PDE5 enzyme, causing an increase in cGMP levels in the corpus cavernosum. Sildenafil increases the cGMP concentration in corporeal smooth muscles with this effect and strengthens the relaxant effect of nitric oxide. The NO - cGMP pathway may also be responsible for vasodilation in the brain through similar mechanisms [1]. PDE-1 and PDE-2 enzymes are involved in cerebral vascularity and sildenafil is also suggested to affect these enzymes [2]. Sildenafil has many side effects shown in the literatüre. The most common side effects are headaches, flushing and nasal congestion caused by vasodilation. Dyspepsia, respiratory infection, dizziness and visual and retinal disturbances are other side effects that can be observed. Changes in pulmonary blood flow indicates the presence of a systemic vasodilation induced by this drug $[3,4]$.

Spontaneous (atraumatic) primary $\mathrm{ICH}$ is a destructive form of stroke associated with high morbidity and mortality. Intracerebral hemorrhage mortality is about $40 \%$ in the first 30 days, and this aspect is similar to subarachnoid hemorrhage in the acute period [5].

Blood flow to the brain vessels increases during sexual intercourse. Sildenafil also increases blood flow to brain vessels, the relationship between sildenafil and intracranial bleeding can be established in this way. It is known that sexual intercourse creates a hyperdynamic circulation state in both men and women due to increased heart rate and blood pressure $[6,7]$. It is also known that sildenafil acts on phosphodiesterase-1, -2 and -5 receptors. This act leads to a secondary increase in intracerebral circulation and vasodilator effects and ultimately leads to sympathetic overactivity, which increases the risk of intracranial bleeding $[8,9]$.

About fourteen cases of adult intracranial hemorrhage associated with the use of PDE-5 were found on review of the English language literature. Cases were between 33 and 70-years-old and our patient was 54-years-old. All these subjects developed cerebral hemorrhage symptoms (including headache, dizziness, blurry vision, decreased mentation, and nausea) and our patient was admitted to the emergency department in a confusional state. In the literatüre in cases of intracranial bleeding with the use of PDE inhibitors, the onset of symptoms was observed within 30 minutes to 3 hours with drug intake. In our case, there was a history of $100 \mathrm{mg}$ sildenafil use 3 hours before the emergency application. In the English literature, none of 14 cases who developed intracranial hemorrhage due to PDE-5 inhibitor use, had a history of intracranial bleeding. The presence of arteriovenous malformation was detected in only one patient, and it was thought that it may be the cause of intracranial hemorrhage. There were no vascular malformations in other reported cases. Bleeding localizations varied considerably from cortex to subcortical areas, and no relationship was found with the age of those cases. The drug doses used by the cases varied between the normal range and overdoses and the relationship between dose and intracranial hemorrhage remains uncertain [10].

\section{Conclusion}

Consequently, high-dose sildenafil use may increase the risk of intracranial bleeding during sexual intercourse by causing cerebral vasodilation, although its relationship with the dose has not been clearly demonstrated. In the previous literature, we did not find any relationship between drug dose $(25 \mathrm{mg}, 50$ $\mathrm{mg}, 100 \mathrm{mg}$ ) and intracranial hemorrhage. However, all subjects used doses of $50 \mathrm{mg}$ or more once or twice in a short period of time. There may be an individual, unknown sensitivity to the drug. Probably, the mode of drug action in large part, may be not cumulative but idiosyncratic. It is extremely important to discuss the risks and benefits before prescribing such drugs to patients with possible underlying vasculopathy and/or previous bleeding. If it is necessary to use these drugs, it may be safer to use them in the lowest possible doses.

\section{Conflict of Interest}

None declared.

\section{References}

1. McHugh J, Cheek DJ (1998) Nitric oxide and regulation of vascular tone: Pharmacological and physiological considerations. Am J Crit Care 7: 131-140.

2. Ballard SA, Gingell CJ, Tang K, Turner LA, Price ME, et al. (1998) Effects of sildenafil on the relaxation of human corpus cavernosum tissue in vitro and on the activities of cyclic nucleotide phosphodiesterase isozymes. J Urol 159: 2164-2171.

3. Morales A, Gingell C, Collins M, Wicker PA, Osterloh IH (1998) Clinical safety of oral sildenafil citrate (VIAGRA) in the treatment of erectile dysfunction. Int $\mathrm{J}$ Impot Res 10: 69-73.

4. Morgan JC, Alhatou M, Oberlies J, Johnston KC (2001) Transient ischemic attack and stroke associated with sildenafil (Viagra) use. Neurology 57: 1730-1731.

5. Aguilar MI, Brott TG (2011) Update in intracerebral hemorrhage. Neurohospitalist 1: 148-159.

6. Abramson DH, Rollins IS, Lin A, Odell P, Folberg R (2006) Tadalafil-induced subretinal and choroidal hemorrhage in a patient with an unsuspected uveal (choroidal and ciliary body) melanoma. Arch Ophthalmol 124: 1058-1060.

7. Kaneria MV, Pagar S, Samant H, Yeole S, Patil S (2008) Subarachnoid haemorrhage: possibly caused by the illegitimate use of sildenafil citrate. J Assoc Physicians India 56: 809-811. 
8. Adiga A, Edriss $H$, Nugent $K$ (2016) Intracranial aneurysm and sildenafil. Proc (Bayl Univ Med Cent) 29: 178-180.

9. Xue-Rui T, Ying L, Da-Zhong Y, Xiao-Jun C (2008) Changes of blood pressure and heart rate during sexual activity in healthy adults. Blood Press Monit 13: 211-217.

10. Lucchese SA, Dhaliwal AS, Kaur A, Qi L (2019) A Case of Recurrent Lobar Intracerebral Hemorrhage in the Setting of Phosphodiesterase-5 Inhibitor Use. Mo Med 116: 400-403. 